Case Report

\title{
Chronic Lymphocytic Leukemia/Small Lymphocytic Lymphoma Presenting as Chronic Diarrhea; An Unusual Presentation of a Common Lymphoproliferative Disorder
}

\author{
Smitha Narayana Gowda (iD, ${ }^{1}$ Hafez Mohammad Abdullah $\left(\mathbb{D}^{1}{ }^{1}\right.$ Rakshya Sharma, ${ }^{1}$ \\ and Mohamed A. Abdallah ${ }^{2}$ \\ ${ }^{1}$ Department of Internal Medicine, University of South Dakota Sanford School of Medicine, Sioux Falls, SD, USA \\ ${ }^{2}$ Department of Pulmonary Medicine, University of South Dakota Sanford School of Medicine, Sioux Falls, SD, USA \\ Correspondence should be addressed to Smitha Narayana Gowda; smithan.1691@gmail.com
}

Received 4 May 2020; Revised 31 March 2021; Accepted 20 April 2021; Published 27 April 2021

Academic Editor: Haruhiko Sugimura

Copyright (C) 2021 Smitha Narayana Gowda et al. This is an open access article distributed under the Creative Commons Attribution License, which permits unrestricted use, distribution, and reproduction in any medium, provided the original work is properly cited.

\begin{abstract}
Chronic lymphocytic leukemia (CLL) is the most common lymphoproliferative disorder in the United States. It has a variable presentation with most patients having asymptomatic lymphocytosis. Many other patients present with lymphadenopathy or enlargement of other organs of the reticuloendothelial system. However, CLL can present with extramedullary involvement. Most commonly, this is in the form of skin or central nervous system involvement, though rarely it can present with gastrointestinal involvement. Here, we present the case of a 70-year-old Caucasian male who presented with chronic diarrhea for over 4 months. After failing conservative treatment, a colonoscopy was performed which showed diffuse mucosal nodularities with a biopsy revealing CLL. The patient was treated successfully with chemotherapy and his diarrhea improved. This is a differential to keep in mind in patients with chronic diarrhea, once the more common causes have been ruled out.
\end{abstract}

\section{Introduction}

B-cell chronic lymphocytic leukemia (CLL) is the most common form of leukemia in the United States, accounting for almost 25 to $30 \%$ of all leukemias [1]. CLL is a progressive accumulation of functionally inert lymphocytes in the blood, lymphoid tissues, and bone marrow. It most commonly affects elderly patients with a median age of diagnosis being 70 years of age [2]. The presentation of CLL can be very variable ranging from asymptomatic lymphocytosis to painless lymphadenopathy, hepatomegaly, splenomegaly, cytopenias, and infections. Patients can also sometimes present with typical B-symptoms such as fever, night sweats, and unintentional weight loss. Sometimes, CLL can transform into a more aggressive form of large B-cell lymphoma called Richter's transformation which can occur in up to $10 \%$ of CLL patients and is associated with poor prognosis.
Extramedullary involvement of CLL is rare, but if it occurs, it most commonly involves the skin and central nervous system [3]. While gastrointestinal involvement can occur rarely in patients with CLL, it mostly occurs in patients with Richter's transformation and is very unusual to occur in patients with CLL [4].

\section{Case Presentation}

A 70-year-old Caucasian male presented with complaints of diarrhea for the last 4 months. He had associated fecal urgency and incontinence at times. He was having 4-5 bowel movements a day ranging from semisolid to watery stools. He denied any history of fevers, abdominal pain, vomiting, weight loss, night sweats, or skin rashes. He denied any recent travel history or exposure to anyone with similar symptoms. 
On physical examination, his abdomen was soft and nontender. There was no apparent hepatomegaly or splenomegaly. The rest of the cardiovascular, respiratory, and nervous system examinations were unremarkable. Initial laboratory evaluation showed a hemoglobin of $15.5 \mathrm{~g} /$ $\mathrm{dL}(14-18)$, WBC of $8.3 \mathrm{~K} / \mathrm{uL}$ (4.5-11), leucocyte fraction of $45.7 \%(15-50 \%)$, platelet count of $187 \mathrm{~K} / \mathrm{uL}(140-440)$, creatinine of $0.9 \mathrm{mg} / \mathrm{dL}(0.7-1.3)$, AST of $34 \mathrm{U} / \mathrm{L}(13-39)$, ALT of $25 \mathrm{U} / \mathrm{L}$ (4-33), ALP of $102 \mathrm{U} / \mathrm{L}$ (34-104), albumin of $4 \mathrm{~g} / \mathrm{dL}$ (3.5-5.7), and globulin of $3.1 \mathrm{~g} / \mathrm{dL}$ (1.5-4.5).

Further workup was done for finding the etiology of the chronic diarrhea. Work up for infection, including parasitic infection and bacterial infections including Clostridium difficile, was negative. He underwent colonoscopy for further evaluation. This revealed a normal mucosa but diffuse submucosal nodularity through the large intestine (Figure 1). Biopsies were taken from multiple sites, and histopathology of the biopsied revealed multiple aggregates for small mature lymphocytes in the submucosa (Figure 2). There was no large cell transformation. Histopathology was negative for CMV colitis. Immunohistochemical stains revealed neoplastic lymphocytes positive for PAX5 (Figure 3), BCL-2, dim BCL-6, CD5, partial CD20, and CD23 and negative for CD3, cyclin D1 (Figure 4), and CD10. A diagnosis of chronic lymphocytic leukemia/small lymphocytic lymphoma was made.

The patient then underwent CT chest, abdomen, and pelvis with IV contrast for evaluation which revealed extensive bilateral axillary, hilar, abdominal, and pelvic lymphadenopathy. He subsequently underwent bone marrow biopsy histopathology which revealed hypercellular bone marrow, with 50\% involved by lymphoid cells; flow cytometric immunophenotyping studies revealed monoclonal B-cell lymphoid population with kappa light chain restriction with CD5 and CD23 coexpression consistent with CLL. The patient did not have any abnormal cell clone harboring deletion of $11 \mathrm{q}, 13 \mathrm{q}$, or $17 \mathrm{p}$. His Rai staging was Stage I and Binet staging was Stage B, putting him at intermediate risk. The patient was referred to oncology that started him on obinutuzumab and venetoclax chemotherapy for the CLL.

The patient went into remission from his CLL on treatment with chemotherapy. The patient improved significantly, and the diarrhea resolved. On 3- and 6-month follow-ups, the patient experienced significant symptomatic improvement with resolution of chronic diarrhea.

\section{Discussion}

A review of the literature revealed a total of $n=5$ reported cases including ours, where the initial presentation of CLL/ SLL was chronic diarrhea [5-8]. This is presented in Table 1. While GI manifestation can occur in CLL, especially in advanced stages or during blast transformation, diarrhea as the initial presentation is rare. In the existing case reports with similar presentation, the mean age of presentation ranged from 65 to 81 years. Associated symptoms were fecal urgency, abdominal pain, and weight loss. One patient presented with abdominal pain and hematochezia and was found later to have intussusception. The duration of symptoms ranged from 4 weeks to 6 months. Three patients had no history of CLL, and one patient had history of CLL that was treated and was thought to be in remission.

Endoscopic evaluation with colonoscopy was notable for variable findings ranging from polypoid mass, multiple linear ulcerations, and ulcerations with mucosal inflammation and friability. Imaging with contrast enhanced CT in all cases revealed abdominal and pelvic lymphadenopathy. Colonic histopathology in all cases revealed lymphocytic infiltration. Treatment details were available for two patients; polypectomy was done for one patient, and rituximab was used to treat for the other patient with resultant resolution of symptoms. In the remaining two patients, one patient decided to pursue comfort care measures and passed away shortly after hospital discharge. No treatment details were available for the other patient who also passed away few weeks after diagnosis.

Of these cases, there are two cases of CCL involving the large intestine including our patient [8]. A case of 66-yearold male who came in whose initial presentation of CLL was abdominal pain and hematochezia and was found to have intussusception on abdominal ultrasound. On further evaluation with colonoscopy, the patient was found to have intraluminal mass in the hepatic flexure. Our patient on the other hand presented with diarrhea and colonoscopy revealed submucosal nodularity in the large intestine. Histopathology of both patients revealed lymphocytic infiltrates. The patient who presented with the colonic mass died within days of diagnosis [7]. Our patient received chemotherapy and is currently in remission.

\section{Additional Points}

(1) Chronic lymphocytic leukemia (CLL) is the most common lymphoproliferative disorder in the United States. (2) It has a very variable presentation with most patients having asymptomatic lymphocytosis. (3) CLL can also present with extramedullary involvement, most commonly the skin or central nervous system. (4) Rarely, it can also present with chronic diarrhea, which typically resolves after treatment of the CLL.

\section{Disclosure}

The abstract of this case report was presented at the American College of Gastroenterology Annual 2020 Conference and the abstract was published in The American Journal of Gastroenterology October 2020 Volume 115. 


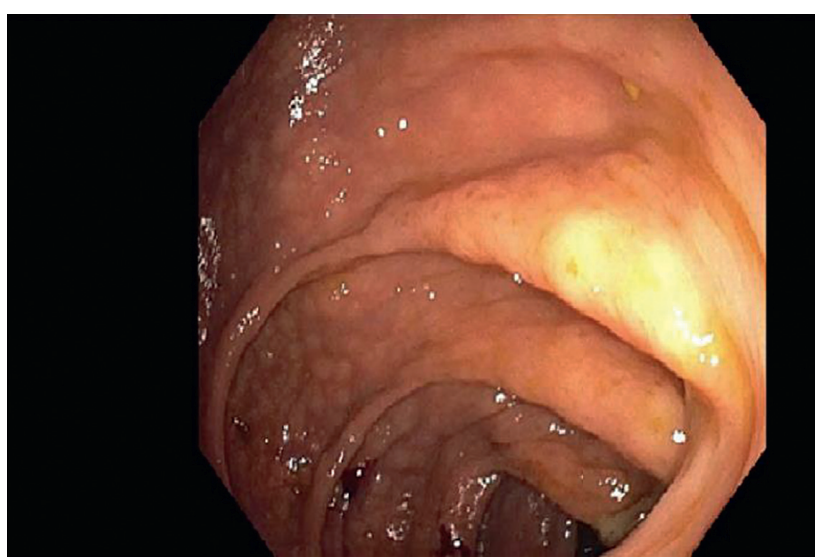

(a)

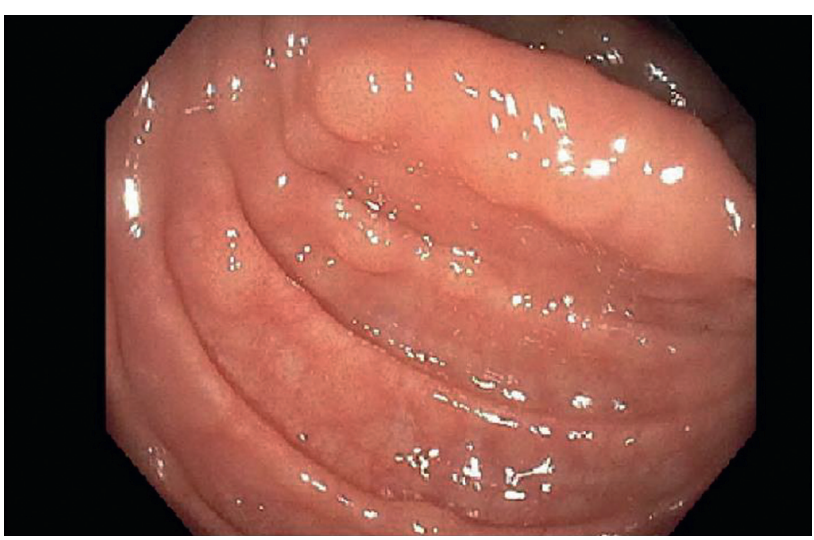

(b)

FIGURE 1: Colonoscopy showing submucosal nodularity.

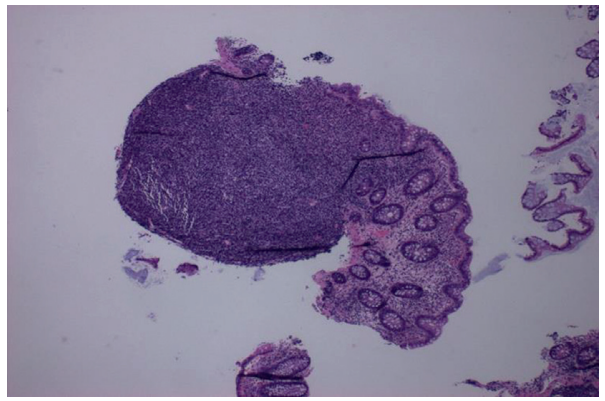

FIGURE 2: Hematoxylin and eosin stain showing lymphoid aggregate with normal colon mucosa.

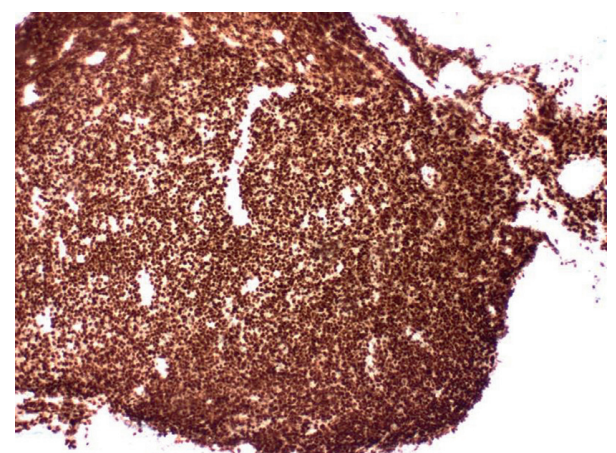

FIgURE 3: Lymphoid aggregate positive for PAX5 stain.

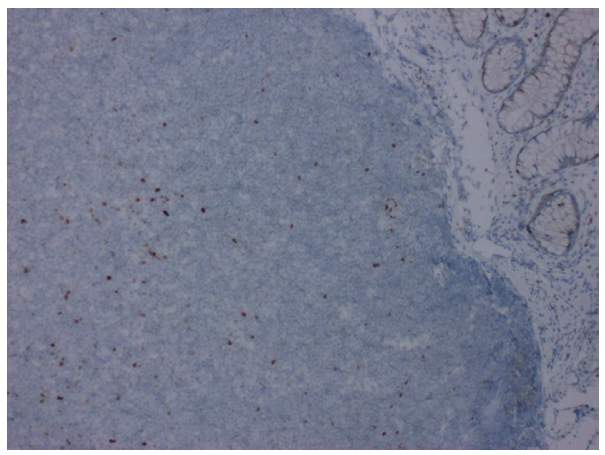

FIGURE 4: Lymphoid aggregate negative for cyclin D1. 


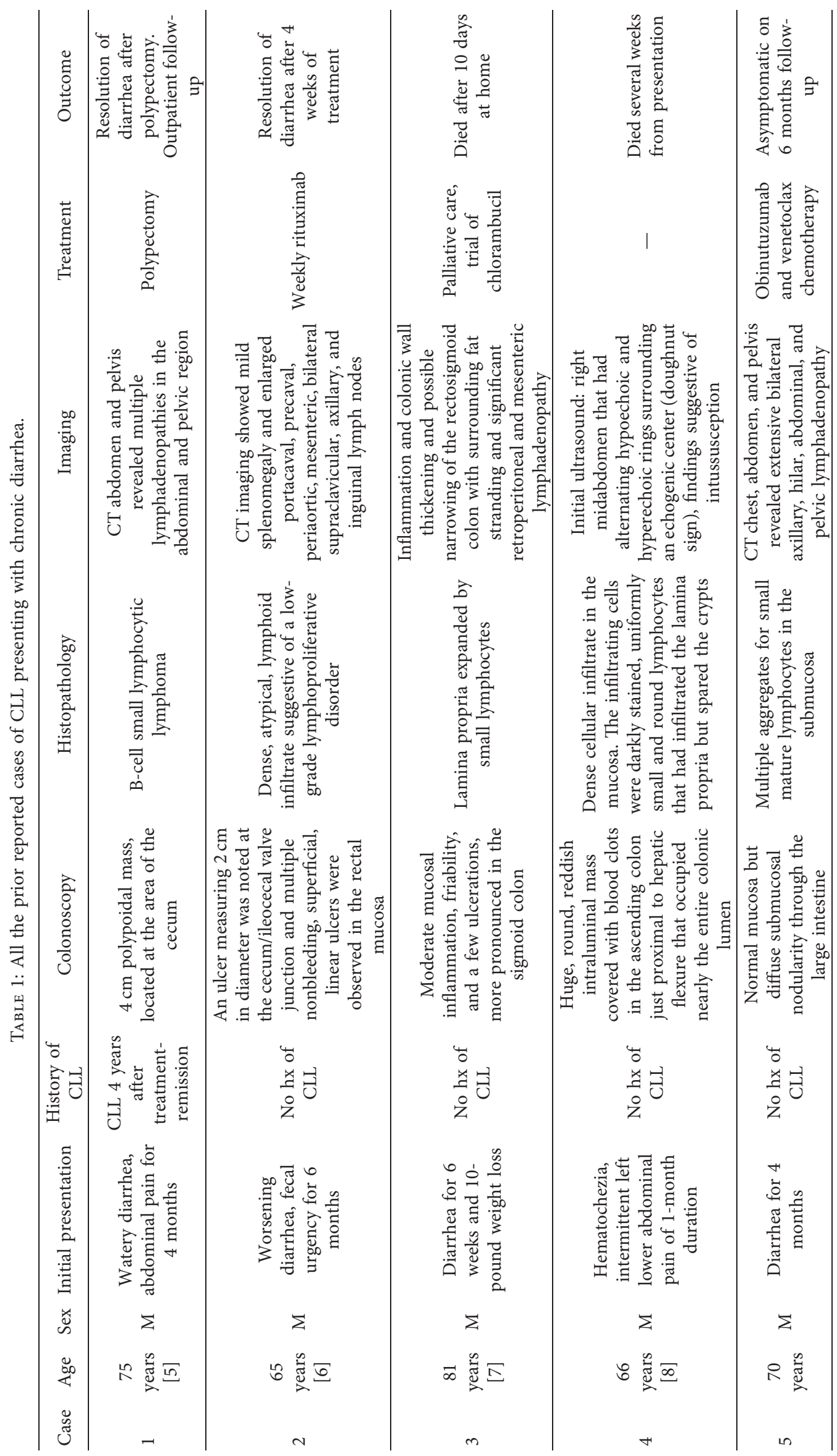




\section{Conflicts of Interest}

The authors declare that they have no conflicts of interest.

\section{References}

[1] R. L. Siegel, K. D. Miller, and A. Jemal, "Cancer statistics, 2020," CA: A Cancer Journal for Clinicians, vol. 70, no. 1, pp. 7-30, 2020.

[2] K. R. Rai and P. Jain, "Chronic lymphocytic leukemia (CLL)then and now," American Journal of Hematology, vol. 91, no. 3, pp. 330-340, 2016.

[3] M. Ratterman, K. Kruczek, S. Sulo, T. D. Shanafelt, N. E. Kay, and C. Nabhan, "Extramedullary chronic lymphocytic leukemia: systematic analysis of cases reported between 1975 and 2012," Leukemia Research, vol. 38, no. 3, pp. 299-303, 2014.

[4] P. E. Arkkila, H. Nuutinen, F. Ebeling, E. Elonen, P. Kärkkäinen, and M. L. Karjalainen-Lindsberg, "Colonic involvement in a patient with chronic lymphocytic leukaemia," Gastroenterology Research and Practice, vol. 2008, Article ID 74214, 4 pages, 2008.

[5] Y. Tuna, E. Koçak, S. Köklü, and İ. Yüksel, "An unusual cause of chronic diarrhea in a patient with chronic lymphocytic leukemia," Southern Medical Journal, vol. 103, no. 5, pp. 487-488, 2010.

[6] C. Xie, K. Aloreidi, and J. Gilbert, "Chronic diarrhea caused by chronic lymphocytic leukemia and small lymphocytic lymphoma with colorectal involvement," Clinical Gastroenterology and Hepatology, vol. 16, no. 3, pp. A33-A34, 2018.

[7] T. A. Zikos, G. Triadafilopoulos, C. Berube, and K. A. Regalia, "Blood and guts: diarrhea from colonic involvement in chronic lymphocytic leukemia/small lymphocytic lymphoma," Digestive Diseases and Sciences, vol. 64, no. 2, pp. 345-348, 2019.

[8] C.-S. Shim, J.-O. Kim, Y. K. Cheon, J. Y. Cho, J. S. Lee, and M. S. Lee, "A case of chronic lymphocytic leukemia-complicated colonic intussusception," Gastrointestinal Endoscopy, vol. 54, no. 1, pp. 77-78, 2001. 